\title{
Comportamentos Agressivos de Crianças na Escola: Evidência de Validade Interna da EPPCACE
}

\author{
Célia Maria Cruz Marques Chaves ${ }^{1}$ \\ Ana Paula Rodrigues Cavalcanti ${ }^{\top}$ \\ Edneia de Oliveira Alves' \\ Karla Santos Mateus' \\ Andrei Alves de Aguiar ${ }^{2}$ \\ Patrícia Nunes da Fonsêca' \\ ${ }^{1}$ Universidade Federal da Paraíba (UFPB), João Pessoa, PB \\ ${ }^{2}$ Núcleo de Pesquisa em Desenvolvimento Sócio-Moral (NPDSM) da UFPB, João Pessoa, PB
}

\begin{abstract}
Resumo
O objetivo do presente estudo foi verificar se os padrões psicométricos da Escala de percepção, por professores, dos comportamentos agressivos de crianças na escola (EPPCACE) evidenciavam sua validade interna. No Estudo $1(n=210)$, foi realizada a adaptação do instrumento e executada uma análise fatorial exploratória. No Estudo $2(n=203)$, efetuou-se uma análise fatorial confirmatória. Participaram professores do ensino fundamental de escolas públicas e particulares. Esses responderam à EPPCACE e a perguntas sociodemográficas. Os resultados indicaram que o instrumento é composto por três fatores: Agressividade direcionada ao âmbito geral $(a=0,95)$, Comportamentos pró-sociais $(a=0,90)$ e Agressividade direcionada aos professores $(a=0,90)$. Conclui-se que a EPPCACE é válida e fidedigna, podendo auxiliar na avaliação do contexto escolar a partir da percepção do professor acerca do comportamento agressivo dos alunos.

Palavras-chave: agressão, professores, escola, evidência de validade
\end{abstract}

Aggressive Behaviors of Children in School: Evidence of EPPCACE internal validity

\begin{abstract}
The objective of this study was to verify whether the psychometric patterns of the Scale of teachers' perception of aggressive behavior of children in school (EPPCACE) showed evidence of its internal validity. In Study $1(\mathrm{n}=210)$ the instrument was adapted and an exploratory factorial analysis was performed. In Study $2(n=203)$ a confirmatory factorial analysis was performed. Elementary school teachers in public and private schools participated and answered the EPPCACE and sociodemographic questions. Results indicated that the instrument is composed of three factors: Aggressiveness directed to the general scope $(\alpha=0.95)$, Pro-social behaviors $(\alpha=0.90)$, and Aggressiveness directed to teachers $(\alpha=0.90)$. We concluded that the EPPCACE is valid and reliable, and it can help in the evaluation of the school context from the teacher's perception of the aggressive behavior of the students.
\end{abstract}

Keywords: aggression; teachers; school; internal validity.

Coductas Agresivas de Niños en la Escuela: Evidencias de validez interna de la EPPCACE

\section{Resumen}

El objetivo del presente estudio fue comprobar si los patrones psicométricos de la Escala de percepción de los maestros sobre el comportamiento agresivo de los niños en la escuela (EPPCACE) evidencian su validez interna. En el estudio 1 ( $\mathrm{n}=210)$ se realizó la adaptación del instrumento y se ejecutó un análisis factorial exploratorio. En el estudio $2(\mathrm{n}=203)$ se efectuó un análisis factorial confirmatorio. Participaron maestros de Enseñanza Primaria de escuelas públicas y privadas. Estos respondieron a la EPPCACE y preguntas sociodemográficas. Los resultados indicaron que el instrumento está compuesto por tres factores: agresividad dirigida al ámbito general $(\alpha=0,95)$, comportamientos pro-sociales $(\alpha=0,90)$ y agresividad dirigida a los maestros $(\alpha=0,90)$. Se concluye que la EPPCACE es válida y fidedigna, pudiendo auxiliar en la evaluación del contexto escolar a partir de la percepción del maestro sobre el comportamiento agresivo de los alumnos.

Palabras clave: agresión; maestros; escuela; evidencias de validez

\section{Introdução}

Diante de mudanças sociais, políticas e econômicas relacionadas a questões como o papel da mulher no mercado de trabalho, a visão e representação sobre a infância e as novas formas de organização familiar, a criança tem sido inserida no sistema educacional brasileiro cada vez mais cedo. A expansão do tempo da criança na escola (Rucinski, Brown, \& Downer, 2017) para atender essas e outras demandas é evidenciada a partir da criação das escolas em tempo integral - regulamentada na LDB 9.394/96 (Brasil, 1996) e pela meta 6 no Plano Nacional de Educação (2014-2024) amparada pela Lei no. 13.005/14 - recentemente aprovada (Brasil, 2014). 
Nesse contexto, os modelos de educação infantil e fundamental foram se modificando na medida em que a concepção de educação para essa faixa etária buscou objetivos diferenciados, sobretudo, respaldados por propostas de trabalho fundamentadas em teorias psicológicas sobre o desenvolvimento infantil (Vokoy \& Pedroza, 2005). Por conseguinte, cada vez mais pesquisas têm se debruçado na verificação dos possíveis ajustes sobre o papel da família e da escola quanto à educação das crianças.

Observa-se, por exemplo, o interesse em averiguar o quanto a família (Renzaho, Silva, \& Sanagorski, 2014) e a escola (Bierman et al., 2013; Bierman, Kalvin, \& Heinrichs, 2015; Elkins, Fite, Moore, Lochman, \& Wells, 2014; He, Koot, Buil, \& Lier, 2017; Masi et al., 2017; Vasconcelos, 2017) contribuem para o desenvolvimento social, emocional e para a redução de problemas de comportamento na infância, como a agressividade. No que se refere especificamente ao contexto escolar, no entanto, mais pesquisas precisam ainda ser realizadas a fim de, por exemplo, apontar estratégias inovadoras sobre como a escola pode agir ativamente na construção de fatores de proteção para condutas de risco (como os comportamentos agressivos em crianças) (Vasconcelos, 2017; Vinha \& Tognetta, 2014; Wang \& Dishion, 2011).

Pesquisas recentes, considerando tanto o contexto internacional quanto nacional (Abenavoli, Greenberg, \& Bierman 2017; Alcantara et al., 2016; Almeida, Fofonka, \& Weiss, 2017; Bierman et al., 2013; Deschamps, Verhulp, Castro, \& Matthys, 2018; Henneberger, Coffman, \& Gest, 2017; Souza 2017; Vieira et al., 2010), indicam que a escola atual é um espaço que abriga vários tipos de agressão e sua incidência entre as crianças tem aumentado significativamente, sendo apontada tanto por professores quanto por outros agentes escolares como uma das maiores dificuldades que enfrentam em sua atuação profissional no contexto escolar (Tavares \& Menin, 2015).

$\mathrm{Na}$ pesquisa desenvolvida no Brasil por Tavares e Menin (2015, com 10.000 participantes no total), os professores apontaram alta frequência de alunos que aborrecem/constrangem uns aos outros $(79 \%)$; agridem-se gritando $(60 \%)$ e são humilhados por diferenças sexuais $(28 \%)$. Nessa mesma direção, o estudo de Carroll, McCarthy, Houghton, O'Connor e Zadow (2018), desenvolvido na Austrália, indicou uma presença expressiva de comportamentos agressivos entre os alunos na escola, sendo: $58,1 \%$ comportamentos de agressão pró-ativa (ação intencional, planejada e coercitiva) física; 57,3\% de agressão pró-ativa verbal; $45,2 \%$ de agressão reativa (ação em resposta à provocação percebida) física e 23,7\% de agressão reativa verbal. Aqui no Brasil, Souza (2017) apontou que, na percepção dos professores, a agressão verbal e física são os tipos de comportamentos agressivos mais comumente observados no contexto escolar.

Deschamps, Verhulp, Castro e Matthys (2018) indicaram que os professores da cidade de Utrecht, na Holanda percebiam o "prazer", a "vontade de chefiar para obter coisas" e a intenção de "ser mau" como explicações possíveis para o comportamento agressivo pró-ativo, e a resposta à provocação, o sentimento de raiva ou o fato de sentir-se ameaçado como fatores de explicação para o comportamento agressivo reativo. Já nos estudos de Abenavoli, Greenberg e Bierman (2017) realizado nos Estados Unidos, observou-se que os professores atribuíam a baixa habilidade socioemocional e moderado engajamento para aprendizagem como fatores relacionados à presença de comportamentos agressivos nos alunos.

Assim, diante dessa realidade brevemente descrita, pensar e favorecer um contexto escolar que vise contribuir para a construção de uma sociedade solidária e promotora de paz (Downes \& Cefai, 2016; UNESCO, 2016) pode ser relevante para um desenvolvimento infantil saudável, principalmente, com as crianças que vivem em situações familiares conflituosas. Nesses casos, a escola atuaria como um agente compensador, na medida em que seria um espaço de construção de habilidades sociais que subsidiariam sua adaptação social em outros contextos (Barna \& Barna, 2014; Reeves $\&$ Brock, 2018).

Diante disso, é importante refletir sobre como professores, diretores, coordenadores e gestores escolares podem atuar a fim de detectar, prevenir e até mesmo inibir comportamentos agressivos entre as crianças na escola. As estratégias mais comuns para atingir esses objetivos, ainda são a punição severa ou ridicularização, no entanto, ao invés de aturem como inibidoras da agressão, contribuem para gerar um acentuado crescimento de hostilidade e alienação na infância (Hanratty, Macdonald, \& Livingstone, 2015; Vinha \& Tognetta, 2014). Tal como discute Grusec (2011), o desafio da atuação voltada para o controle comportamental é adotar estratégias de intervenção sem favorecer o aparecimento de problemas internos (culpa, depressão) ou externos (expressão de comportamentos de raiva). Desse modo, as pesquisas voltadas ao domínio do controle comportamental buscam verificar como as normas 
ou regras de socialização podem ser internalizadas pelas crianças a fim de que não ocorram prejuízos na autonomia e promovam a autorregulação comportamental (Grusec, 2011).

Uma vez que a criança não consegue compreender a "importância da norma” ou construir tal compreensão de forma significativa, o respeito às normas sociais fica consideravelmente comprometido e o avanço no desenvolvimento da moralidade estacionado em níveis iniciais, como atestam diferentes pesquisadores e teóricos na área da Psicologia, baseados, sobretudo, nas contribuições de Kolberg, Piaget e Hoffman (ver revisão realizada por Galvão, 2010). Não avançar no processo de desenvolvimento moral, por sua vez, também pode ser um dos indicadores relacionados a problemas futuros, como aumento de comportamentos agressivos (Borsa \& Bandeira, 2011; Souza \& Castro, 2008).

Crianças que se comportam de forma agressiva apresentam, em maior frequência, baixo rendimento escolar e um marcador de risco para consequências, como uso de drogas e delinquência juvenil (Bierman et al., 2013; Bierman et al., 2015; Elkins et al., 2014; He et al., 2017; Masi et al., 2017).

Em contrapartida, Gonçalves e Murta (2008) afirmam que crianças com amplo repertório de habilidades sociais (avanço na construção do desenvolvimento moral, empatia, assertividade e autocontrole, dentre outras) conseguem estabelecer relações sociais mais saudáveis, expondo-se a um risco menor de rejeição pelos pares. Estas ainda apresentam um fator de proteção contra dificuldades de aprendizagem e comportamentos agressivos. Andrade, Fernandes e Ferreira (2017) indicaram ainda que pode haver uma relação entre omissão do professor e relação conflituosa entre os alunos e que o professor pode ser um agente decisivo na redução desses conflitos.

O padrão comportamental da díade professor-aluno na pré-escola mostrou, em uma série de estudos longitudinais realizados na Bélgica, que a qualidade do relacionamento emocional e o nível de conflito dessa díade podem prever o grau de agressividade adotado pela criança na escola (Verschueren, Cadima, \& Doumen, 2014). Nessa mesma direção, outras pesquisas (Gest, Madill, Zadzora, Miller, \& Rodkin, 2014; Rucinski et al., 2017) apontam que o rendimento escolar tende a melhorar conforme o relacionamento professor-aluno torna-se mais positivo. Resultados desse tipo revelam a necessidade de estudos de levantamento para averiguação da relação entre a percepção do professor e o comportamento agressivo dos alunos para, então, serem tomados como base de políticas públicas educacionais de prevenção e redução da agressividade na escola.

Apesar dos altos índices de agressividade no contexto escolar mencionados nas pesquisas empíricas relatadas até aqui, poucos são os instrumentos psicológicos produzidos no Brasil para atender a essa demanda (Borsa \& Bandeira, 2011). No tocante a realidade brasileira, em levantamento que considerou publicações em um período de 20 anos, Borsa e Bandeira (2011) indicaram que, dentre os poucos instrumentos de pesquisa que visam avaliar a agressão infantil na escola, figura a EPPCACE desenvolvida por Lisboa e Koller (2001). Os itens do referido instrumento foram desenvolvidos com a participação de juízes, pois se tratava de uma validação qualitativa, que utilizou a técnica de análise de conteúdo e, logo, a publicação não trouxe informações psicométricas que indicassem evidências de sua validade interna.

Dentre as definições possíveis de comportamento agressivo, Lisboa e Koller (2001) adotaram a ideia de que esse comportamento se refere a "toda ação que causa ou implica danos ou prejuízos a alguém, e é expresso de forma confrontativa e/ou não confrontativa" (p. 60). Assim, para a construção da EPPCACE, Lisboa e Koller (2001) consideraram duas perspectivas de análise diretamente relacionadas: os tipos de agressão e a direção do comportamento agressivo. No tocante aos tipos de agressão, observaram as práticas confrontativas (a atos diretos sejam eles físicos ou verbais) e não confrontativas (a atos hostis indiretos). Já considerando a direção da agressão, adotaram aquelas dirigidas aos professores, colegas e/ou para um âmbito geral. Incluíram também 15 itens relativos a comportamentos positivos ou pró-sociais de crianças no contexto escolar afim de "despistar" o participante acerca do construto que a escala avaliava, visando evitar o viés no foco da investigação. Assim, ao final do processo de análise de conteúdo obtiveram uma escala composta por 41 itens: 26, relativos a afirmações de comportamentos agressivos e 15, sobre comportamentos positivos.

É diante dessa realidade, que se justifica a importância da presente pesquisa tanto para a produção de conhecimento na Psicologia quanto por sua relevância social. Logo, objetivou-se verificar se os padrões psicométricos da EPPCACE evidenciavam sua validade interna e, considerando a escassez de instrumentos de avaliação da agressão no contexto e público em que essa pesquisa se insere, espera-se contribuir para a redução dessa lacuna observada. Para tanto, foram desenvolvidos dois estudos com amostras independentes utilizando, 
no primeiro, a análise de validade fatorial exploratória da medida e, no segundo, a validade confirmatória e convergente descritas a seguir.

\section{Estudo 1. Análise Exploratória da EPPCACE}

O objetivo do Estudo 1 foi verificar as primeiras evidências psicométricas de validade e consistência (alfa de Cronbach) baseadas na estrutura interna da EPPCACE.

\section{Método}

\section{Participantes}

Participaram dessa etapa 210 professores do ensino fundamental de escolas públicas $(55,8 \%)$ e particulares $(44,2 \%)$ da cidade de João Pessoa (PB), com média de idade de 28,35 anos $(D P=8,92)$, sendo a maioria do sexo feminino (87\%) e cursando a graduação em Pedagogia (78,3\%). Tratou-se de uma amostra não probabilística, portanto de conveniência. A participação dos profissionais ocorreu voluntariamente.

\section{Instrumentos}

Os participantes responderam a um questionário formado por duas partes:

EPPCACE (Lisboa \& Koller, 2001). Composta por 41 itens, avalia a percepção dos professores acerca dos comportamentos dos alunos na escola. $\mathrm{Na}$ parte superior, antes da apresentação dos itens da escala, estão dispostas instruções informando aos participantes que os itens do instrumento devem ser respondidos considerando uma escala de cinco pontos, variando de $1=$ Discordo totalmente a $5=$ Concordo totalmente.

Informações Demográficas. Organizaram-se oito perguntas de natureza demográfica a fim de caracterizar os participantes do estudo nas seguintes variáveis: sexo, idade, estado civil, escolaridade, curso de graduação, período que está cursando, tempo que exerce a profissão e tipo de escola em que leciona (pública ou privada).

\section{Procedimentos de Coleta e Análise de Dados}

A presente pesquisa está ligada a um projeto mais amplo, aprovado pelo Comitê de Ética em Pesquisa do Centro de Ciências da Saúde da Universidade Federal da Paraíba (CAAE 39013714.0.0000.5188), respeitando a Resolução 466/2012 do Conselho Nacional de Saúde, que trata da pesquisa com seres humanos (Brasil, 2012).

Em um primeiro momento, buscou-se verificar se o instrumento era compreensível e não apresentava dificuldades de interpretação. Seguindo as indicações de Pasquali (2010), procedeu-se a validação semântica. Para tanto, consideraram-se participantes da população-alvo: 20 professores, dos quais, 10 com formação superior em andamento e 10 na pós-graduação (mestrado/doutorado). A partir desse processo inicial não foram apontadas e realizadas grandes modificações na redação dos itens da escala, sendo possível seguir com a coleta.

A coleta ocorreu após os participantes assinarem um Termo de Consentimento Livre e Esclarecido (TCLE) e todos os objetivos da pesquisa, o caráter voluntário e possibilidade de desistência, sem danos, serem explicados. Os professores foram abordados tanto em uma Universidade Federal da cidade de João Pessoa, na qual exerciam suas atividades ligadas à graduação, quanto em instituições escolares em que ministravam aulas. A aplicação foi efetuada por colaboradores devidamente treinados que permaneceram à disposição durante todo o tempo de aplicação, que durou em média 20 minutos.

Para a tabulação e análises estatísticas dos dados, foi utilizado o SPSS (Statistical Package for the Social Science, versão 18). Realizaram-se inicialmente as estatísticas descritivas (medidas de tendência central e de dispersão, distribuição de frequência e percentual) para caracterizar a amostra de participantes estudada, teste $t$ de Student, a fim de averiguar o poder discriminativo dos itens. Foi tomado como hipótese nula que os grupos critérios formados pelos participantes que pontuaram abaixo da mediana e o formado pelos que pontuaram acima não apresentariam diferenças estatisticamente significativas, correlação item-total (homogeneidade do fator), como mais uma evidência de precisão da medida, análise de Componentes Principais (APC), buscando identificar a estrutura fatorial preliminar da escala e análise do alfa de Cronbach para aferir a consistência interna da escala. Antes da análise de APC, contudo, visou-se conhecer a adequação da matriz de correlação dos dados coletados, a partir do Kaiser-Meyer-Olkim (KMO) e do Teste de Esfericidade de Bartlett (Tabachnick \& Fidell, 2013). Posteriormente, consideraram-se os critérios de Guttman-Kaiser; Cattell e o cálculo da análise paralela para averiguar o número de componentes a extrair (Laros, 2008). Os resultados são descritos a seguir.

\section{Resultados e Discussão}

Inicialmente, inverteram-se as escalas de respostas dos 15 itens que eram semanticamente contrários à 
maioria daqueles que compunham a escala (Ex., item 13: Ajuda o/a professor/a). Em seguida, o somatório dos 41 itens resultou em uma pontuação total da qual foi calculada a mediana $(M d=90)$ e, a partir dela, foi possível dividir os participantes exatamente em dois grupos critérios, um com pontuação inferior à mediana e outro com pontuação superior. Esse procedimento, seguindo os passos de Pasquali (2010) foi adotado a fim de realizar o teste $t$ de Student de medidas independentes para cada item e identificar se os itens discriminariam as respostas dos participantes com pontuações próximas. Os resultados indicaram que todos os itens apresentaram poder discriminativo na direção esperada $(t>3, p<$ $0,001)$, com isso, prosseguiu-se com as análises contando com o conjunto total de itens. A adequabilidade da realização de uma análise fatorial exploratória na matriz dos dados foi comprovada a partir dos valores do $\mathrm{KMO}=0,94$ e do Teste de Esfericidade de Bartlett significativo $(\chi 2(820)=5822,730, p<0,001)$ (Tabachnick \& Fidell, 2013).

De posse desses resultados, decidiu-se efetuar uma Análise de Componentes Principais (ACP), uma vez que esse método é mais indicado quando o foco é a redução de fatores naqueles que explicam a máxima variância (Hair, Anderson, Tatham, \& Black, 2005). Optou-se ainda por não fixar tipo de rotação ou número de fatores a extrair, uma vez que não houve estudos anteriores que visassem averiguar evidências de validade baseada na estrutura interna da medida. Assim, nessa oportunidade, apresentam-se evidências empíricas preliminares da escala, uma vez que, na pesquisa anterior que tratou da construção da escala as autoras do instrumento (Lisboa \& Koller, 2001), realizaram uma análise de conteúdo, não sendo, até o momento, observadas outras evidências empíricas que indicassem de forma mais consistente, como orientado por Brown (2006), por exemplo, como os itens desse instrumento deveriam se organizar.

Três critérios foram adotados para fundamentar a decisão sobre o número de fatores: a) Guttman-Kaiser, valores próprios iguais ou maiores que 1 (ver, por exemplo, Hair, Black, Babin, Anderson, \& Tatham, 2009); b) Cattell, ou seja, a distribuição gráfica dos valores próprios (Hair et al., 2009) e c) Análise Paralela de Horn (AP), que compara os eigenvalues de uma matriz de correlação de dados aleatórios com os da matriz empírica. Por meio dos resultados da AP, indica-se reter um fator se este explicar maior variância do que aquele correspondente nos dados aleatórios (ver, por exemplo, Laros, 2008; O'Connor, 2000).
De acordo com o critério de Guttman-Kaiser, foram observados seis componentes fatoriais (eigenvalues - valores próprios: 18,38; 2,56; 1,92; 1,42; 1,22 e 1,16), que explicaram conjuntamente $65 \%$ da variância total. A distribuição gráfica (scree plot) desses eigenvalues, observada pelo critério de Cattel, contudo, indicou ser mais adequada uma solução com três fatores. O terceiro critério, AP, foi realizado tendo como referência os 210 participantes e 41 itens, realizando-se 1.000 simulações. Essa análise revelou a existência de, no máximo, três componentes, pois o valor próprio do quarto componente da matriz empírica $(1,42)$ foi inferior ao quarto valor observado na análise paralela $(1,69)$, o que permite não considerá-lo. Dessa forma, assumiu-se a estrutura organizada em três fatores. Dando continuidade, efetuou-se uma nova análise ACP, dessa vez fixando-se a quantidade de três fatores com rotação varimax, tomando em conta para a interpretação da solução fatorial os itens que apresentaram carga fatorial (saturação) acima de $|0,30|$. Os resultados dessa análise são dispostos na Tabela 1.

A partir da organização dos itens conforme apresentação da Tabela 1, os três fatores da EPPCACE podem ser descritos como segue:

Fator I - Agressividade direcionada ao âmbito geral. O primeiro fator reuniu dezessete itens, com saturação variando de 0,50 (Estraga objetos dos(as) colegas) a 0,79 (Implica com os(as) colegas e os (as) provoca). Apresentou valor próprio de 18,38 , explicando $44,83 \%$ da variância total. A consistência interna resultou em um coeficiente de alfa de Cronbach igual a 0,95. Ao realizar uma leitura do conteúdo dos itens que se agruparam nesse fator, decidiu-se denominá-lo de "Agressividade direcionada a um âmbito geral", uma vez que denotam uma ideia de comportamentos agressivos dirigidos a diferentes figuras do convívio escolar (professores, colegas, pais dos colegas) ou que revelam uma característica do sujeito agressor (ex.: item 17 - É uma criança agressiva).

A decisão pela eliminação de itens foi realizada com base nos seguintes critérios: (1) valor absoluto da carga fatorial principal do item menor do que $|0,30|$ (Pasquali, 2010); (2) possuir cargas fatoriais similares em dois ou mais fatores em um mesmo item, com a diferença entre valores absolutos das cargas fatoriais dos itens menor do que 0,10 (Gorsuch, 1983) e (3) ausência de similaridade entre o conteúdo do item e o domínio teórico do construto (Smith \& McCarthy, 1995). Como é possível observar nos resultados dispostos na Tabela 1 , no tocante ao primeiro fator, o critério da análise de juízes (critério 03) auxiliou na decisão de eliminação do 
Tabela 1.

Estrutura Fatorial da EPPCACE

\begin{tabular}{|c|c|c|c|c|c|c|}
\hline Conteúdo dos itens & $\begin{array}{c}\text { Fator } \\
\text { I }\end{array}$ & $\begin{array}{c}\text { Fator } \\
\text { II }\end{array}$ & $\begin{array}{c}\text { Fator } \\
\text { III }\end{array}$ & $h^{2}$ & $\mathrm{r}_{\mathrm{it}}$ & $\alpha_{\text {Del }}$ \\
\hline 36. Implica com os(as) colegas e os (as) provoca. & 0,79 & & & 0,72 & 0,75 & 0,96 \\
\hline 23. Seus colegas reclamam de seu comportamento. & 0,76 & & & 0,63 & 0,83 & 0,95 \\
\hline 24. $\mathrm{O}$ (a) professor(a) também reclama de seu comportamento. & 0,74 & & & 0,67 & 0,77 & 0,96 \\
\hline 35. Ridiculariza os(as) colegas. & 0,74 & & & 0,73 & 0,81 & 0,96 \\
\hline 15. Chuta, bate, morde os(as) colegas. & 0,74 & & & 0,67 & 0,76 & 0,96 \\
\hline 17. É uma criança agressiva. & 0,72 & & & 0,64 & 0,82 & 0,96 \\
\hline 34. Amedronta, intimida os(as) colegas. & 0,72 & & & 0,71 & 0,82 & 0,96 \\
\hline 27. Ameaça os(as) colegas. & 0,70 & & & 0,63 & 0,83 & 0,96 \\
\hline 25. Costuma danificar objetos do ambiente escolar. & 0,69 & & & 0,62 & 0,72 & 0,96 \\
\hline 40. Provoca intrigas entre os(as) colegas. & 0,68 & & & 0,57 & 0,80 & 0,96 \\
\hline $\begin{array}{l}\text { 05. Os pais dos(as) colegas reclamam do comportamento desta } \\
\text { criança com seus filhos. }\end{array}$ & 0,65 & & & 0,50 & 0,66 & 0,96 \\
\hline 14. É uma criança considerada valentona. & 0,63 & & & 0,50 & 0,74 & 0,96 \\
\hline 30. Discute com os(as) colegas. & 0,63 & & & 0,62 & 0,68 & 0,96 \\
\hline 07. Briga com os(as) colegas. & 0,61 & & & 0,45 & 0,68 & 0,96 \\
\hline $\begin{array}{l}\text { 04. É uma criança arrogante e debochada (que faz brincadeiras de } \\
\text { mau gosto). }\end{array}$ & 0,60 & & & 0,48 & 0,68 & 0,96 \\
\hline 28. Fala palavrões. & 0,58 & & & 0,48 & 0,71 & 0,96 \\
\hline 22. Estraga objetos dos(as) colegas. & 0,50 & & & 0,42 & 0,64 & 0,96 \\
\hline 13. Ajuda o(a) professor(a). & & 0,74 & & 0,62 & 0,63 & 0,89 \\
\hline 01. É uma criança cooperativa. & & 0,69 & & 0,59 & 0,61 & 0,89 \\
\hline 16. Tem amigos. & & 0,65 & & 0,49 & 0,61 & 0,89 \\
\hline 37. É gentil com o(a) professor(a). & & 0,65 & & 0,65 & 0,70 & 0,90 \\
\hline 06. Participa em sala de aula. & & 0,64 & & 0,44 & 0,76 & 0,89 \\
\hline 02. Ouve o(a) professor(a). & & 0,61 & & 0,55 & 0,63 & 0,89 \\
\hline 12. Ajuda os(as) colegas. & & 0,59 & & 0,47 & 0,75 & 0,89 \\
\hline 29. Gosta de trabalhar em grupo. & & 0,59 & & 0,41 & 0,68 & 0,89 \\
\hline 32. Preocupa-se com o que é certo e errado. & & 0,57 & & 0,50 & 0,56 & 0,90 \\
\hline 38. Os (as) colegas demonstram gostar dele. & & 0,53 & & 0,45 & 0,70 & 0,89 \\
\hline 03. Ouve os(as) colegas. & & 0,51 & & 0,32 & 0,38 & 0,90 \\
\hline 19. Gosta de brincar em grupo. & & 0,50 & & 0,31 & 0,49 & 0,90 \\
\hline 10. Reconhece quando seus colegas estão chateados. & & 0,47 & & 0,27 & 0,41 & 0,90 \\
\hline 11. Briga com o(a) professor(a). & & & 0,73 & 0,63 & 0,67 & 0,89 \\
\hline 20. Tenta amedrontar, intimidar o(a) professor(a) & & & 0,73 & 0,67 & 0,76 & 0,88 \\
\hline 18. Desafia o(a) professor(a). & & & 0,72 & 0,66 & 0,72 & 0,89 \\
\hline 31. Discute com o(a) professor(a). & & & 0,71 & 0,73 & 0,75 & 0,88 \\
\hline 08. Tenta ridicularizar o(a) professor(a). & & & 0,68 & 0,67 & 0,72 & 0,90 \\
\hline 41. Implica com o(a) professor(a) e o (a) provoca. & & & 0,65 & 0,71 & 0,73 & 0,89 \\
\hline 33. Ameaça o(a) professor(a). & & & 0,60 & 0,65 & 0,64 & 0,90 \\
\hline 26. É uma criança confiável & 0,57 & 0,49 & - & - & - & - \\
\hline 39. Tenta agredir fisicamente o(a) professor(a) & 0,44 & & 0,40 & - & - & - \\
\hline 09. Quando contrariado, nega-se a realizar tarefas. & 0,40 & 0,33 & 0,32 & - & - & - \\
\hline 21. É gentil com os(as) colegas & 0,47 & 0,52 & 0,32 & - & - & - \\
\hline Número de itens & 17 & 13 & 7 & - & - & - \\
\hline Valor próprio & 18,38 & 2,56 & 1,92 & - & - & - \\
\hline Variância Total & 44,83 & 6,24 & 4,68 & - & - & - \\
\hline Alfa de Cronbach $(\alpha)$ & 0,95 & 0,90 & 0,90 & - & - & - \\
\hline
\end{tabular}

Nota. EPPCACE = Escala de percepção, por professores, dos comportamentos agressivos de crianças na escola. $\mathrm{h}^{2}=$ Comunalidade; $\mathrm{r}_{\mathrm{it}}=$ Correlação item-total; $\alpha_{\text {Del }}=$ Alfa de Cronbach se o item for deletado. 
item 26 (É uma criança confiável), que apesar de saturar significativamente no fator mencionado, não demonstra coesão ou relação semântica com os outros itens do componente fatorial. Já a eliminação dos itens 39 (Tenta agredir fisicamente o(a) professor(a)) e 09 (Quando contrariado, nega-se a realizar tarefas) foi realizada com base no segundo critério anteriormente descrito (Gorsuch, 1983), porque tais itens apresentaram saturação significativa e muito próxima em mais de um fator.

Fator II - Comportamentos pró-sociais. O segundo fator reuniu 13 itens, que variaram de 0,47 (Reconhece quando seus colegas estão chateados) a 0,74 (Ajuda o (a) professor(a)). Seu valor próprio foi de 2,56, explicou $6,24 \%$ da variância total e apresentou consistência interna (alfa de Cronbach) de 0,90. A nomeação do fator foi pautada tanto pela leitura do conteúdo dos itens quanto pela classificação proposta por Lisboa e Koller (2001) e Lisboa (2005). Os 13 itens desse fator revelam claramente a ideia da não apresentação de comportamentos agressivos (ex.: item $37-$ É gentil com o(a) professor(a)). No entanto, excluiu-se o item 21 (É gentil com os(as) colegas) por saturar significativamente nos três fatores da escala (Gorsuch, 1983).

Fator III - Agressividade direcionada aos professores. Agruparam-se sete itens, variando de 0,60 (Ameaça o(a) professor(a)) a 0,73 (Briga com o(a) professor(a)), com valor próprio de 1,92 e explicando 4,68\% da variância total. $\mathrm{O}$ valor do alfa de Cronbach revelou alta consistência interna $(a=0,90)$. A denominação do fator foi motivada pelo fato de que todos os itens expressaram claramente diferentes comportamentos agressivos alvejados ao professor. Nesse fator não houve itens eliminados. Considerando a validação de conteúdo realizada por Lisboa e Koller (2001), mencionada anteriormente, observou-se que os itens indicados como representantes das categorias Agressividade direcionada a um âmbito geral e Agressividade direcionada aos(às) colegas se uniram, na presente pesquisa, formando o Fator I (Agressividade direcionada a um âmbito geral); enquanto que as categorias Comportamentos pró-sociais e Agressividade direcionada aos professores praticamente se mantiveram idênticas nos dois contextos, formando, no presente estudo, os fatores II (Comportamentos pró-sociais) e III (Agressividade direcionada aos professores), respectivamente. No que se refere especificamente às formas ou tipos de agressão (confrontativa e não confrontativa), compreende-se que essa é mais uma especificação da natureza da agressão que é direcionada à figura do professor, ao colega ou ainda ao âmbito geral. Com isso, é possível observar, inclusive, que os itens que as representam estão mesclados entre as categorias de direção da agressão (Lisboa \& Koller, 2001). Dessa forma, acredita-se ser mais interessante a comparação dos achados do presente estudo com as categorias de direção da agressão bem como aquela dos comportamentos pró-sociais.

Observou-se, então, que a estrutura da EPPCACE com 37 itens organizados em três fatores, apresenta evidências psicométricas satisfatórias uma vez que os indicadores de validade e precisão descritos para os três fatores são consonantes aos valores recomendados na literatura especializada (Cohen, Swerdlik, \& Sturman, 2014; Laros, 2008; Hair et al., 2009; Pasquali, 2010; Tabachnick \& Fidell, 2013). Em seguida, decidiu-se verificar essa estrutura em um segundo estudo com uma amostra independente por meio de uma análise estatística mais robusta, a análise fatorial confirmatória (AFC) descrita a seguir.

\section{Estudo 2. Confirmação da Estrutura Fatorial da EPPCACE}

O objetivo do Estudo 2 foi avaliar se a estrutura trifatorial revelada no Estudo 1 era adequada para representar os dados empíricos da EPPCACE. Para tanto, realizou-se uma AFC considerando a versão reduzida de 37 itens indicada no Estudo 1.

\section{Método}

\section{Participantes}

Participaram 203 professores do ensino fundamental do estado da Paraíba, com média de idade de 35,30 anos $(D P=9,42)$. Destes, $58,6 \%$ trabalhavam em escolas públicas; $32 \%$ em escolas particulares e 9,4\% lecionavam tanto em escolas públicas quanto particulares. A maior parte dos participantes era do sexo feminino $(78,6 \%)$ e do curso de Pedagogia (53,8\%). Tratou-se de uma amostra não probabilística, tendo participado os profissionais que concordaram em colaborar voluntariamente.

\section{Instrumentos e Procedimentos de Coleta e Análise de Dados}

Os participantes responderam o mesmo questionário apresentado no Estudo 1, que reunia a EPPCACE e perguntas de natureza demográfica. A coleta dos dados foi realizada em instituições escolares em que os participantes ministram aulas e, após consentimento livre e esclarecido, as respostas foram obtidas de forma individual. O tempo médio para concluir a participação foi de aproximadamente 20 minutos. 
Objetivando testar a estrutura trifatorial da versão adaptada da EPPCACE, composta por 37 itens, o software Amos (versão 18) foi utilizado para realização da AFC. Considerou-se como entrada a matriz de covariância, adotando-se o método de estimação Maximum Likelihood (ML). A fim de verificar a qualidade de ajuste do modelo testado, foram levados em consideração múltiplos indicadores de ajuste comumente empregados na literatura (Hair et al,, 2009; Tabachnick \& Fidell, 2013), descritos a seguir.

$\chi^{2}$ (Qui-quadrado). Esse indicador revela a probabilidade do modelo teórico se ajustar aos dados. Valores baixos são considerados mais adequados. No entanto, uma vez que é influenciado pelo tamanho da amostra bem como pelo número de variáveis do modelo, costuma-se adotar sua razão quanto aos graus de liberdade (Razão $\chi^{2} / g h$ ) a fim de obter um indicador mais robusto. Mesmo não existindo um valor crítico exato para decidir se o modelo é ou não adequado, na prática, consideram-se valores entre 2 e 3 , sendo ainda aceitável valores até 5 , para indicar a adequação do modelo teórico.

GFI (Goodness-of-Fit Index) e AGFI (Adjusted Goodness-of-Fit Index). São indicadores análogos ao $\mathrm{R}^{2}$ da regressão múltipla, expressando assim a proporção de variância-covariância nos dados, explicada pelo modelo. O AGFI, entretanto, é ponderado em relação aos seus graus de liberdade. Eles variam de 0 a 1 , aceitando-se como indicadores de ajuste do modelo aqueles próximos ou superiores a 0,90 .

CFI (Comparative Fit Index). É um indicador comparativo adicional de ajuste ao modelo, que apresenta melhor desempenho quando o tamanho da amostra é pequeno (Tabachnick \& Fidell, 2013). Valores próximos ou superiores a 0,90 revelam melhor ajuste do modelo.

RMSEA (Root-Mean-Square Error of Approximation). Avalia o quanto o modelo permite explicar os dados considerando-se os valores residuais. É um dos indicadores de ajuste mais informativo e parcimonioso, pois seleciona o modelo com menor número de parâmetros. Com seu intervalo de confiança de 90\% (IC $90 \%$ ) revela que valores altos indicam um modelo não ajustado. Assim, um valor próximo a zero é indicativo de ajuste do modelo aos dados uma vez que os residuais correspondentes se apresentam mais próximos desse valor. Recomenda-se dessa forma que valores entre 0,05 e 0,08, admitindo-se até 0,10 expressam um ajuste satisfatório.
Além desses indicadores usuais, calcularam-se, a partir dos coeficientes padronizados da AFC, a variância média extraída (VME) e a confiabilidade composta (CC) (Gouveia \& Soares, 2015), que fornecem evidências complementares de validade interna (Hair et al., 2005). Valores iguais ou superiores a 0,50 e 0,70, respectivamente, são identificados na literatura como parâmetros de adequação satisfatórios (Hair et al., 2009). A VME pode ser considerada ainda como um índice de validade convergente (o quanto explica o conjunto de itens) e a CC como adicional da consistência interna. É salutar considerar essa medida uma vez que o alfa de Cronbach é influenciado pelo número de itens (Pasquali, 2010). Apresentam-se a seguir os resultados e discussão dessas análises.

\section{Resultados e Discussão}

A AFC foi empregada sendo observados os seguintes indicadores: razão $X^{2} / g l=2,04 ; \mathrm{GFI}=0,74$; AGFI $=0,71$; CFI: 0,88; RMSEA $=0,07$ (intervalo de confiança de 90\%: 0,08-0,10). Todos os itens da EPPCACE apresentaram saturações (pesos fatoriais, $\lambda)$ estatisticamente significativas e diferentes de zero $(p<0,001)$. A estrutura correspondente é disposta na Figura 1.

Conhecida a estrutura fatorial da medida, procurou-se ainda observar evidências complementares da validade interna (CC e VME), precisão (alfa de Cronbach) e homogeneidade da escala. No que consiste à CC, os valores encontrados para os três fatores (FI: 0,96; FII: 0,90; FIII: 0,90) foram superiores ao recomendado pela literatura $(0,70)$ conforme Hair, Anderson, Tatham e Black (2009). No caso da VME, sua validade convergente ficou também evidenciada para os fatores I $(0,58)$ e III $(0,57)$ (Hair et al., 2009). Por fim, observaram-se correlações médias inter-itens $(0,58)$ e item-total $(0,42)$ as quais revelaram uma medida homogênea. De posse de tais resultados, observa-se que os procedimentos de $A F C$ indicam a adequação do modelo composto por três fatores, conforme observado anteriormente no Estudo 1. Nessa direção, é importante pontuar ainda que o fato dos indicadores de ajuste GFI $(0,74)$ e AGFI $(0,71)$ apresentarem valores razoáveis não representa comprometimento na adequação do modelo, uma vez que, quando analisados em conjunto com os demais indicadores, apóia-se à adequação psicométrica da estrutura sugerida que indica evidências de validade fatorial e de consistência interna. 


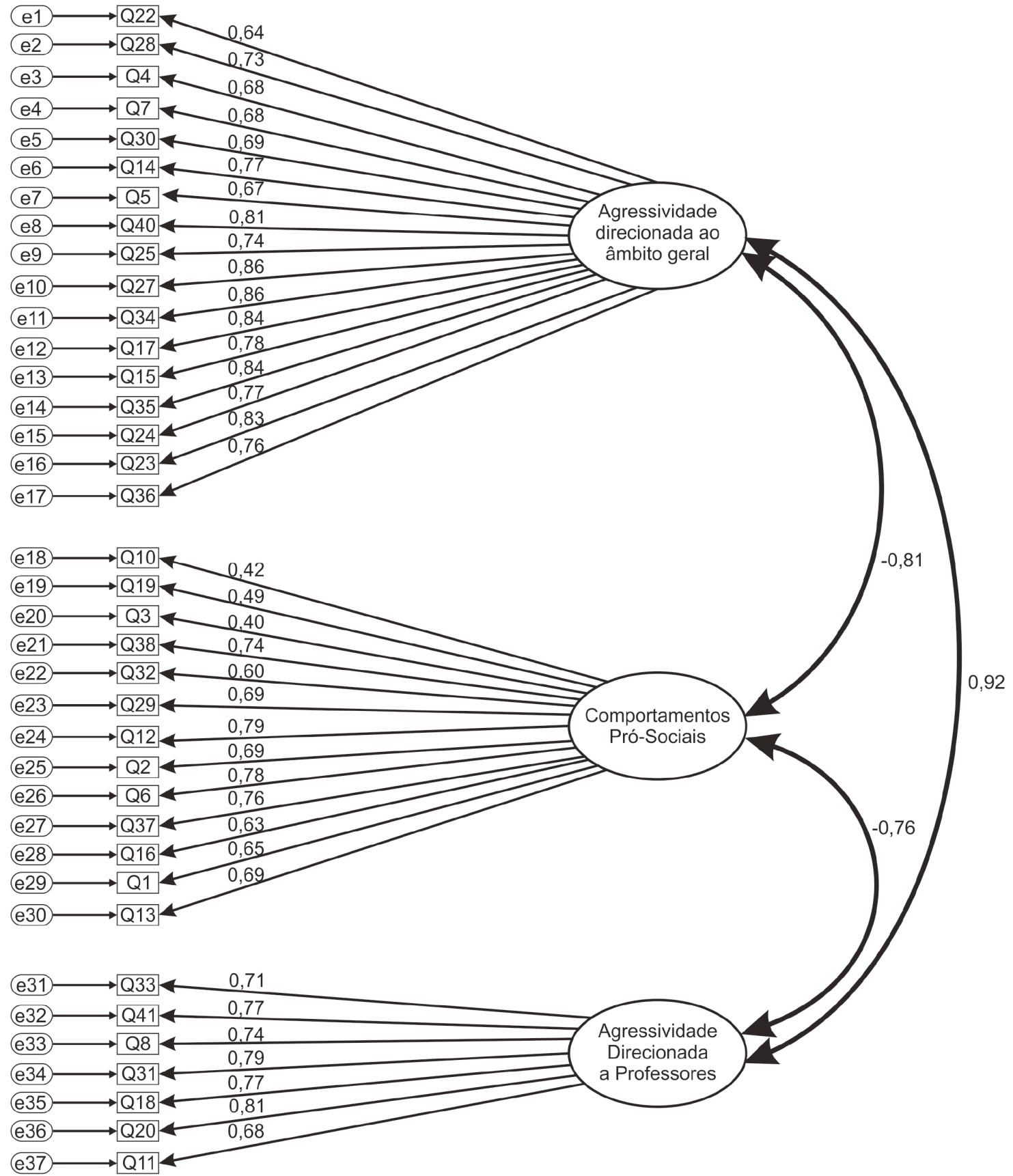

Figura 1. Estrutura fatorial da EPPCACE.

Além desse aspecto, é possível pensar, conforme dispõe Brown (2003), que erros correlacionados podem representar tanto itens com semânticas semelhantes, como eventuais dificuldades de leitura de itens invertidos e até particularidades de demanda. E, mesmo que a proximidade semântica não seja suficiente para explicar as correlações, é possível assumir que alguma relação não especificada pode também influenciar essa associação.

\section{Discussão Geral e Considerações Finais}

O objetivo geral da presente pesquisa foi verificar se os padrões psicométricos da EPPCACE evidenciavam 
sua validade interna. Os resultados obtidos permitiram atingir tal objetivo e indicar que a versão reduzida com 37 itens estruturados em três fatores é pertinente para a avaliação da percepção, por professores, sobre o comportamento agressivo de crianças na escola, tal como supunham Lisboa e Koller (2001).

Observou-se que o primeiro fator esteve representado pelos itens das categorias I e II descritas por Lisboa e Koller (2001), e o segundo e terceiro fatores, respectivamente, pelas categorias III e IV (Lisboa \& Koller, 2001). No entanto, quatro itens não integraram a versão final da escala, o que resultou em uma versão reduzida de 37 itens.

A AFC (Estudo 2), por sua vez, confirmou a estrutura trifatorial, observando indicadores de ajuste aceitáveis, que se aproximaram daqueles preconizados na literatura como satisfatórios (Hair et al., 2009; Tabachnick \& Fidell, 2013). No tocante à consistência interna, tanto os alfas de Cronbach quanto os valores de confiabilidade composta (CC) estavam acima dos valores comumente recomendados na literatura $(0,70)$ conforme descrevem Pasquali (2010) e Hair et al. (2009). Reforçando a estabilidade da EPPCACE, as correlações inter-itens indicaram a homegeneidade da medida, o que auxilia na evidência de sua adequação.

A breve revisão de literatura descrita anteriormente aponta que a escola tem sido um cenário fértil para a expressão de comportamentos agressivos entre as crianças de diferentes contextos culturais (Abenavoli et al., 2017; Deschamps, et al., 2018; Carroll et al., 2018; Souza, 2017; Tavares \& Menin, 2015) trazendo prejuízos consideráveis ao seu desenvolvimento e aprendizagem das crianças (Bierman et al., 2015; Elkins et al., 2014; Masi et al., 2017), como também dificultado consideravelmente a atuação profissional dos agentes educacionais na escola (Tavares \& Menin, 2015).

Assim, contar com um instrumento que possa ser utilizado para identificar "os tipos de agressão" presentes entre os alunos no contexto escolar, pode ser útil no delineamento de ações de docentes, gestores escolares e pessoas da família no sentido de observar com mais cuidado quais os fatores podem estar relacionados à presença de comportamentos agressivos pelos alunos a fim de propor estratégias de intervenção que visem diminuir ou quiçá sanar os problemas presentes e futuros geralmente relacionados aos comportamentos agressivos, como também contribuir para o fomento de políticas públicas educacionais de prevenção e redução da agressividade na escola.
Apesar de tais resultados, é possível elencar limitações na presente pesquisa. Considerando o objetivo dos dois estudos desenvolvidos, a utilização de duas amostras independentes, com pouco mais de 200 participantes, respectivamente, possibilita a realização de análises estatísticas robustas que corroboraram a qualidade psicométrica da EPPCACE (Pasquali, 2010), contudo, na expansão das conclusões para além do seu escopo figura limitações. Em estudos futuros, pode ser pertinente considerar amostras de professores e professoras mais amplas e diversificadas, por exemplo, quanto à região geográfica (outras capitais e cidades interioranas) e nível de formação (graduação e pós-graduação).

Outra limitação recai sobre o fato do instrumento ser direcionado aos professores. Essa avaliação do comportamento dos alunos pode ser relevante, no entanto, contar apenas com a percepção desse profissional pode não fornecer dados que de fato se aproximem da realidade, pois a subjetividade do professor pode enviesar sua avaliação do comportamento das crianças. Assim, em estudos futuros pode ser apropriado realizar a adaptação dos itens da EPPCACE a fim de serem respondidos pelos próprios alunos e, dessa forma, poder contar com mais um olhar sobre a questão, possibilitando também comparar a percepção do professor com a autopercepção do estudante.

Além disso, é relevante conhecer evidências de validade de critério (concorrente e, principalmente, preditiva) e estabilidade temporal (teste-reteste). Assim, por exemplo, poderia ser avaliada em que medida uma alta pontuação na EPPCACE explicaria a dificuldade de concentração, aprendizagem e de adaptação escolar (Borsa \& Bandeira, 2011) ou ainda em que medida a postura de professores e diretores no sentido de punir ou ridicularizar tais comportamentos agressivos dos alunos podem contribuir para a presença de mais hostilidade e alienação (Vinha \& Tognetta, 2014; Hanratty et al., 2015). Quanto à estabilidade temporal (Pasquali, 2010), é preciso avaliar a magnitude de flutuação com o tempo ou eventos históricos identificados dessa medida.

O manuseio desse instrumento é algo muito favorável, considerando a simplicidade de suas instruções, da escala de resposta e do número relativamente pequeno de itens. Nesse sentido, confia-se que a EPPCACE contribua para o levantamento mais objetivo e sistemático de informações sobre a percepção de professores a cerca de comportamentos agressivos de crianças na escola e assim, colabore com Borsa e Bandeira (2011), no tocante a escassez de medidas com essa finalidade. 
É importante enfatizar, no entanto, que o presente instrumento, na forma como se apresenta nesse artigo de validação, ainda não pode ser utilizado para fins de diagnóstico. Tal como se observa na literatura especializada, outros estudos que visem, por exemplo, o estabelecimento de normas locais ou nacionais de referência (Anastasi \& Urbina, 2000; Pasquali, 2010) precisam ser efetuados quando houver essa intenção diagnóstica. A normatização de um teste permite assim que os escores produzidos por ele sejam comparados e contextualizados (Pasquali, 2010), ajustando à realidade do pesquisador, clínico e/ou educador.

\section{Referências}

Abenavoli, R. M., Greenberg, M. T., \& Bierman, K. L. (2017). Identification and validation of school readiness profiles among high-risk kindergartners. Early Childhood Research Quarterly, 38, 33-43. doi: 10.1016/j.ecresq.2016.09.001

Alcantara, S., González-Carrasco, M., Montserrat, C., Viñas, F., Casas, F., \& Abreu, D. P. (2016). Peer violence in the School Environment and Its Relationship with Subjective Well-Being and Perceived Social Support Among Children and Adolescents in Northeastern Brazil. Journal of Happiness Studies, 18, 1507-1532. doi: 10.1007/s10902-016-9786-1

Almeida, L. D., Fofonka, L., \& Weiss, C. S. (2017). Violência, ética, moral e bullying no espaço escolar. Revista Maiêutica - Estudos Linguísticos, Literários e Formação Docente, 5(1), 53-62. Recuperado de https://publicacao.uniasselvi.com.br/index.php/ LED_EaD/article/view/1751/859

Anastasi, A., \& Urbina S. (2000). Testagem psicológica. Porto Alegre: Editora Artes Médicas.

Andrade, M. S., Fernandes, J. S. G., \& Ferreira, V. S. (2017). Estudo sobre a relação professor-aluno. Revista de Pós-Graduação Multidisciplinar, 1(2), 335-346. doi: 10.22287/rpgm.v1i2.601

Barna, I., \& Barna, O. (2014). The effectiveness of school-based violence prevention programs for reducing aggressive behavior. Procedia - Social and Behavioral Sciences 137, 88 - 92. doi: 10.1016/j. sbspro.2014.05.257

Bierman, K. L., Coie, J., Dodge, K., Greenberg, M., Lochman, J., McMahon, R., Pinderhughes, E., \& TheCPPRG. (2013). School outcomes of aggressive-disruptive children: Prediction from kindergarten risk factors and impact of the Fast Track Prevention Program. Aggressive Behavior, 39, 114-130. doi: 10.1002/ab.21467

Bierman, K. L., Kalvin, C. B., \& Heinrichs, B. S. (2015). Early childhood precursors and adolescent sequelae of grade school peer rejection and victimization. Journal of Clinical Child and Adolescent Psychology, 44(3), 367-379. doi: 10.1080/15374416.2013.873983

Borsa, J. C., \& Bandeira, D. R. (2011). Uso de instrumentos psicológicos de avaliação do comportamento agressivo infantil: Análise da produção científica brasileira. Avaliação Psicológica, 10(2), 193 203. Recuperado de http://www.redalyc.org/ articulo.oa?id $=335027286010$

Brasil. Lei n. 13.005, de 24 de junho de 2014. Aprova o Plano Nacional de Educação - PNE e dá outras providências. Recuperado de http://www.planalto.gov.br/ccivil_03/_Ato2011-2014/2014/Lei/ L13005.htm

Brasil, MINISTÉRIO DA SAUDE. Conselho Nacional de Saúde. RESOLUÇÃO No 466, de 12 de dezembro de 2012. Recuperado de http:// bvsms.saude.gov.br/bvs/saudelegis/cns/2013/ res0466_12_12_2012.html

Brasil. Lei n. 9.394, de 20 de dezembro de 1996. Estabelece as Diretrizes e Bases da Educação Nacional. Diário Oficial da União, Brasília, 23-12-96.

Brown, T. A. (2003). Confirmatory fator analysis of the penn state worry questionnaire: Multiple factors or method effects? Behaviour Research and Therapy, 41(12), 1411-1426. doi: 10.1016/ S0005-7967(03)00059-7

Brown, T. A. (2006). Confirmatory factor analysis for applied research. New York: The Guilford Press.

Carroll, A., McCarthy, M., Houghton, S., O'Connor, E. S., \& Zadow, C. (2018). Reactive and proactive aggression as meaningful distinctions at the variable and person level in primary school-aged children. Aggressive Behavior. (1-11). doi: 10.1002/ab.21763

Cohen, R. J., Swerdlik, M. E., \& Sturman, E. D. (2014). Testagem e avaliação psicológica: Introdução a testes e medidas ( $8^{\circ}$ ed). São Paulo: AMGH.

Deschamps, P. K., Verhulp, E. E., de Castro, B. O., \& Matthys, W. (2018). Proactive aggression in early school-aged children with externalizing behavior 
problems: A longitudinal study on the influence of empathy in response to distress. American Journal of Orthopsychiatry. Advance online publication. doi: 10.1037 /ort0000319

Downes P., \& Cefai, C. (2016) How to Prevent and Tackle Bullying and School Violence: Evidence and Practices for Strategies for Inclusive and Safe Schools, NESET II Report, Luxembourg: Publications Office of the European Union. doi: 10.2766/0799

Elkins, S. R., Fite, P. J., Moore, T. M., Lochman, J. E., \& Wells, K. C. (2014). Bidirectional effects of parenting and youth substance use during the transition to middle and high school. Psychology of Addictive Behaviors, 28(2), 475-486. doi: 10.1037/a0036824

Galvão, L. K. de S. (2010). Desenvolvimento moral e empatia: Medidas, correlatos e intervenções educacionais (Tese de doutorado não publicada). Recuperado de http://bdtd.biblioteca.ufpb.br/ bitstream/tede/7012/1/arquivototal.pdf

Gest, S. D., Madill, R. A., Zadzora, K. M., Miller, A. M., \& Rodkin, P. C. (2014). Teacher management of elementary classroom social dynamics: Associations with changes in student adjustment. Journal of Emotional and Behavioral Disorders, 22(2), 107-118. doi: $10.1177 / 1063426613512677$

Gonçalves, E. S., \& Murta, S. G. (2008). Avaliação dos efeitos de uma modalidade de treinamento de habilidades sociais para crianças. Psicologia Reflexão e Crítica, 21(3), 430-436. Recuperado de http: // www.scielo.br/pdf/prc/v21n3/v21n3a11

Gorsuch, R. L. (1983). Factor analysis (2a ed). New York: McGraw-Hill.

Gouveia, V. V., \& Soares, A. K. S (2015). Calculadora de validade de construto (CVC). João Pessoa, PB: BNCS/ Universidade Federal da Paraíba.

Grusec, J. E. (2011). Socialization processes in the family: Social and emotional development. Annual Review of Psychology, 62(1), 243-269. doi: 10.1146/ annurev.psych.121208.131650

Hair, J. F., Anderson, R. E., Tatham, R. L., \& Black, W. C. (2005). Análise multivariada de dados. Porto Alegre: Artmed.

Hair, J. F., Black, W. C., Babin, B. J., Anderson, R. E., \& Tatham, R. L. (2009). Análise multivariada de dados. (6 $\left.6^{\mathrm{a}} \mathrm{Ed}\right)$. Porto Alegre: Artmed.
Hanratty, J., Macdonald, G., \& Livingstone, N. (2015). Child-focused psychosocial interventions for anger and aggression in children under 12 years of age. Cochrane Database of Systematic Reviens, 7. doi: 10.1002/14651858.CD011788

He, J., Koot, H. M., Buil, J. M., \& Lier, P. A. C. (2017). Impact of low social preference on the development of depressive and aggressive symptoms: Buffering by children's prosocial behavior. Journal of Abnormal Cbild Psychology, 19. Advance online publication. doi: 10.1007/s10802-017-0382-6

Henneberger, A. K., Coffman, D. L., \& Gest, S. D. (2017). The effect of having aggressive friends on aggressive behavior in childhood: Using propensity scores to strengthen causal inference. Social Development, 26(2), 295-309. doi: 10.1111/sode.12186

Laros, J. A. (2008). O uso da análise fatorial: Algumas diretrizes para pesquisadores. Em: Pasquali, L. (Ed.). Análise Fatorial Para Pesquisadores (p. 181-202). Brasília: LabPAM, UnB.

Lisboa, C. S. M. (2005). Comportamento agressivo, vitimização e relações de amizade de crianças em idade escolar: fatores de risco e proteção (Tese de doutorado não publicada). Recuperado de http://hdl.handle. net/10183/6875

Lisboa, C. S. M., \& Koller, S. H. (2001). Construção e validação de conteúdo de uma escala de percepção, por professores, dos comportamentos agressivos de crianças na escola. Psicologia em Estudo, 6(1), 5969. doi: 10.1590/S1413-73722001000100008

Masi, G., Manfredi, A., Nieri, G., Muratori, P., Pfanner, C., \& Milone, A. (2017). A naturalistic comparison of methylphenidate and risperidone monotherapy in drug-naive youth with attentiondeficit/hyperactivity disorder comorbid with oppositional defiant disorder and aggression. Journal of Clinical Psychopharmacology, 37(5), 590-594. doi: 10.1097/ JCP.0000000000000747

O'Connor, B. P. (2000). SPSS and SAS programs for determining the number of components using parallel analysis and velicer's MAP test. Behavior Research Methods 32(3), 396-402. doi: 10.3758/ BF03200807

Pasquali L. (2010). Instrumentação Psicológica: Fundamentos e Práticas. Porto Alegre: Artmed. 
Reeves, M. A. L., \& Brock, S. E. (2018). Responding to students who threaten violence: Helping handout for the school [Handout]. Bethesda, MD: National Association of School Psychologists. Recuperado de https:// www.nasponline.org/Documents/Resources $\% 20$ and $\% 20$ Publications/Resources/Crisis/Threat_ Assessment_Reeves.Brock_2018_Rev.pdf

Renzaho, A. M. N., \& Silva-Sanagorski, A. (2014). The importance of family functioning, mental health and social and emotional well-being on child oral health. Child: care, health and development, 40(4), pp.543-552. doi: 10.1111/cch.12053

Rucinski, C. L., Brown, J. L., \& Downer, J. T. (2017). Teacher-Child Relationships, Classroom Climate, and Children's Social-Emotional and Academic Development. Journal of Educational Psychology. (113). Advance online publication. doi: 10.1037/ edu0000240

Smith G. T., \& McCarthy D. M. (1995). Methodological considerations in the refinement of clinical assessment instruments. Psychol Assess, 7(3), 300-308. doi:10.1037/1040-3590.7.3.300

Souza, M. A., \& Castro, R. E. F. de (2008). Agressividade infantil no ambiente escolar: Concepções e atitudes do professor. Psicologia em Estudo, 13(4), 837-845. doi: 10.1590/S1413-73722008000400022

Souza, S. B. B. (2017). A influência da violência na interação professor-aluno. Campos dos Goytacazes (RJ). (Trabalho de Conclusão de Curso em Licenciatura em Ciências Sociais). Recuperado de https://app.uff.br/riuff/handle/1/5024

Tabachnick, B. G., \& Fidell, L. S. (2013). Using multivariate statistics ( $6^{\mathrm{a}}$ ed.). New York: Allyn \& Bacon.

Tavares, M. R., \& Menin, M. S. S. (2015). Avaliando valores em escolares e seus professores: Proposta de construção de uma escala. São Paulo: Textos FCC.
UNESCO (2016). Global Education Monitoring Report. Unesco: Paris, France. Recuperado de http://unesdoc.unesco.org/images/0024/002469/246984e. pdf

Vasconcelos, I. C. O. (2017). Aprender a conviver, sem violência: $\mathrm{O}$ que dá e não dá certo? Ensaio: Avaliação e Politicas Públicas em Educação, 25(97), 897-917. Epub August 21, 2017. doi: 10.1590/ S0104-40362017002501180

Verschueren, K., Cadima, J., \& Doumen, S. (2014). De rol van leerkracht-kind interacties in deontwikkeling van probleemgedrag tijdens de transitie naar de lagere school [The role of teacherstudent interactions in the development of problem behavior during the transition to primary school]. Pedagogische Studiën, 91(5), 318-331. Recuperado de https:// www.researchgate.net/publication/304015831

Vieira, L. J. E. S., Abreu, C. A. P., Valdês, M. T. M., Oliveira, E. N., Ferreira, R. C., \& Catrib, A. M. F. (2010). Violência na escola pública: Relatos de professores. RBPS Fortaleza, 23 (1), 34-42. doi: 10.7476/9788575413302

Vinha, T., \& Tognetta, L. R. P. (2014). Os conflitos interpessoais no Brasil e as violências escondidas. International Journal of Developmental and Educational Psychology, 7(1), 323-331. doi: 10.5020/1169

Vokoy, T., \& Pedroza, R. L. S. (2005). Psicologia escolar em educação infantil: Reflexões de uma atuação. Psicologia Escolar e Educacional, 9(1), 95-104. doi: 10.1590/S1413-85572005000100009

Wang, M.-T., \& Dishion, T. J. (2011). The trajectories of adolescents' perceptions of school climate, deviant peer affi liation, and behavioral problems during the middle school years. Journal of Research on Adolescence, 22(1), 40-53. doi: 10.1111/j.1532-7795.2011.00763.x

Recebido em: $27 / 11 / 2017$ Reformulado em: 24/05/2018; 13/08/2018

Aprovado em: 29/08/2018 
Sobre os autores:

Célia Maria Cruz Marques Chaves é doutora em Psicologia Social pela Universidade Federal da Paraíba (UFPB), professora adjunta do Departamento de Psicopedagogia da UFPB e integrante do núcleo de pesquisa em Desenvolvimento Sócio-Moral (NPDSM). Seus interesses de pesquisa centram-se nas áreas de Psicologia, com ênfase em Psicologia do Desenvolvimento Humano, Comportamentos agressivos, Processos de Socialização Materna, Síndrome de Down, Educação e Inclusão.

ORCID: https://orcid.org/0000-0002-3852-0226

E-mail: celliachaves@yahoo.com.br

Ana Paula Rodrigues Cavalcanti é doutora em Psicologia Social pela Universidade Federal da Paraíba (UFPB), professora adjunta do Departamento de Ciências das Religiões da UFPB e integrante do núcleo de pesquisa Videlicet, na linha de pesquisa de espiritualidade e saúde, assim como o grupo de pesquisa Liepas, da UniRio, na mesma temática. Possui como áreas de interesse Psicologia, Teologia, Espiritualidade e Saúde.

ORCID: https://orcid.org/0000-0003-4128-5885

E-mail: anapaulacavalcanti.ufpb@gmail.com

Edneia de Oliveira Alves: é licenciada em Letras (português e inglês) pela Universidade Federal de Pernambuco, mestre e doutora em Psicologia Social, professora de Libras (Língua Brasileira de Sinais) da Universidade Federal da Paraíba, professora da Pós-Graduação em Letras - PPGL da UFPB e coordena os projetos de extensão e de pesquisa sobre material visual para ensino ao surdo.

ORCID: https://orcid.org/0000-0001-6645-1419

E-mail: edneiaalvesufpb@gmail.com

Karla Santos Mateus: é mestra em Psicologia Social pela Universidade Federal da Paraíba (UFPB), atualmente, doutoranda do Programa de Pós-Graduação em Psicologia Social (UFPB) e colaboradora do Grupo de Pesquisa em Comportamento Político (GPCP), com áreas de interesse centradas em Psicologia Social, Comportamento político, Preconceito e Racismo.

ORCID: https://orcid.org/0000-0001-5398-9578

E-mail: karlasmateus@gmail.com

Andrei Alves de Aguiar: é psicólogo, mestre em Ciências das Religiões e doutor em Psicologia Social, pela UFPB, membro pesquisador do Grupo NOUS - Espiritualidade e Sentido e do Núcleo de Pesquisa em Desenvolvimento Sócio-Moral (NPDSM). Possui trabalhos com a temática do Sentido da vida, Bem-estar subjetivo, Religiosidade, Perdão Interpessoal e Normatividade.

ORCID: https://orcid.org/0000-0002-4495-4581

E-mail: andreilogoterapia@gmail.com.

Patrícia Nunes da Fonsêca é graduada em Psicologia pela Universidade Federal da Paraíba, doutora em Psicologia Social pela UFPB, docente da Pós-graduação em Psicologia Social e do Departamento de Psicopedagogia da UFPB e, atualmente, atua também como professora da Educação a distância do curso de Pedagogia da UFPB. Interessa-se por temáticas relacionadas ao desenvolvimento infantil, família e psicologia jurídica.

ORCID: https://orcid.org/0000-0002-6322-6336

E-mail: patynfonseca@hotmail.com

Contato com os autores:

Célia Maria Cruz Marques Chaves

Universidade Federal da Paraíba, CE, Departamento de Psicopedagogia

João Pessoa-PB, Brasil

CEP: 58051-900 